\title{
Efficient Oxidation of Adamantanes by Sodium Nitrite with Molecular Oxy- gen in Trifluoroacetic Acid
}

\author{
Osamu Onomura, ${ }^{\mathrm{a}}$ Yutaka Yamamoto, ${ }^{\mathrm{a}}$ Noriaki Moriyama, ${ }^{\mathrm{a}}$ Fumiaki Iwasaki ${ }^{\mathrm{b}}$ and Yoshihiro \\ Matsumura*a \\ “a Graduate School of Biomedical Sciences, Nagasaki University, 1-14 Bunkyo-machi, Nagasaki 852-8521, Japan” \\ “'b Tsukuba Research Lab., Tokuyama Co. Ltd., 40 Wadai, Tsukuba 300-4247, Japan”
}

Fax: $+81-95-819-2476$

E-mail: matumura@net.nagasaki-u.ac.jp

Received: The date will be inserted once the manuscript is accepted.

Abstract: Oxidation of adamantanes by oxygen was effectively achieved by use of sodium nitrite as a catalyst in trifluoroacetic acid (TFA) to give 1-adamantyl trifluoroacetates, from which adamantanols were obtained in good yields. A catalytic amount of sodium nitrite was essential and TFA was the best solvent among solvents examined for this oxidation.

Key words: Alcohols, Hydrocarbons, Oxidations, Oxygen, Radical Reactions.

Oxidation of adamantane (1a) to 1-adamantanol (3a) has continued to attract much interest because of a versatility of 3a in material sciences. ${ }^{1}$ Among a variety of methods for the transformation of $\mathbf{1 a}$ to $\mathbf{3 a}$ or its derivatives, ${ }^{2}$ use of oxygen as an oxidant is worthwhile from both economical and mechanistic viewpoints; iron(III)/aldehyde $/ \mathrm{O}_{2},{ }^{3} \quad$ iron(II) $/ \mathrm{H}_{2} \mathrm{~S} / \mathrm{O}_{2},{ }^{4} \quad \mathrm{NHPI} / \mathrm{O}_{2},{ }^{5}$ $\mathrm{TiO}_{2} / \mathrm{hv} / \mathrm{O}_{2}{ }^{6}$ electrochemical reduction/Tl(III)/porphyrin $/ \mathrm{O}_{2}{ }^{7}$ Ru(III)-polyoxometalate $/ \mathrm{O}_{2}{ }^{8}{ }^{8}$ and $\mathrm{V}(\mathrm{IV})$-polyoxometalate $/ \mathrm{O}_{2}{ }^{9}$. However, those reported methods give 3a in low yields and require some metal ion or organic halide catalyst under drastic conditions. We report herein an efficient method for oxidation of adamantanes 1a-c to 1-adamantanols 3a-c using a catalytic amount of easily available sodium nitrite $\left(\mathrm{NaNO}_{2}\right)$ with molecular oxygen (Scheme 1).

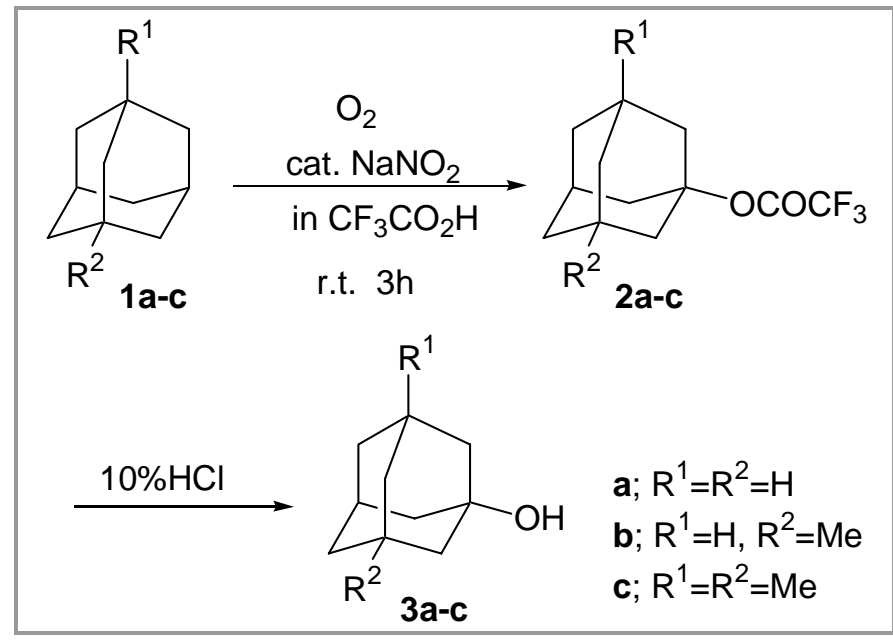

Scheme 1
A typical condition of our method is as follows; a solution of $1 \mathbf{a}$ in TFA containing 0.2 equiv of $\mathrm{NaNO}_{2}$ was stirred at r.t. under an oxygen atmosphere. Since the organic extract of the reaction was a mixture of 1adamantyl trifluoroacetate (2a) and a few \% of $\mathbf{3 a}$, it was then treated with an aqueous $1 \mathrm{~N} \mathrm{HCl}$ solution overnight followed by extraction with $\mathrm{CH}_{2} \mathrm{Cl}_{2}$ to afford $\mathbf{3 a}$ in $96 \%$ yield without contamination of $\mathbf{2 a}$. Similar reaction of methyl-substituted adamantanes $\mathbf{1 b}, \mathbf{c}$ with oxygen afforded methyl-substituted adamantanols 3b,c in good yields. Those results were shown in Table 1 (Entries 3-5) In order to give some insight into the reaction mechanism, oxidation of 1a-c was carried out under a variety of reaction conditions (Entries 1, 2, 6-17 in Table 1).

The results indicated that three kinds of factors were critical for the oxidation of 1a-c to 3a-c. First one is the effect of $\mathrm{NaNO}_{2}$ as a catalyst on the yield of 3a. That is, the absence of $\mathrm{NaNO}_{2}$ didn't cause any oxidation of $\mathbf{1 a}$ (Entry 1 ) but only $1 \mathrm{~mol} \%$ of $\mathrm{NaNO}_{2}$ was enough for the completion of the oxidation of $\mathbf{1 a}$ (Entry 2). $\mathrm{NOBF}_{4}{ }^{10}$ was usable in placement of $\mathrm{NaNO}_{2}$ (Entry 6), whereas $\mathrm{NaNO}_{3}$ didn't cause any oxidation of 1a (Entry 7).

The second one was an oxygen effect. The reactions in Entries 1-7 were carried out under an oxygen atmosphere (1 atm) or an aerobic condition where satisfactory results were obtained when $\mathrm{NaNO}_{2}$ was used as a catalyst (Entries $2-5,8)$. However, the yield of 3a was narrowly $10 \%$ under a nitrogen atmosphere even if 0.2 equiv of $\mathrm{NaNO}_{2}$ was used (Entry 9). Another noticeable point was that the yields of 3a were dependent on the amount of $\mathrm{NaNO}_{2}$ (46\% for 1 equiv of $\mathrm{NaNO}_{2}$; Entry 10, and $92 \%$ for 2 equiv of $\mathrm{NaNO}_{2}$; Entry 11).

The third one was a solvent effect. $\mathrm{AcOH}$ and $\mathrm{MsOH}$ were not suitable to the oxidation (Entries 12,13) but a prolonged reaction time gave $\mathbf{3 a}$ in a satisfactory yield in the latter solvent (Entry 14). Chlorodifluoroacetic acid was a usable solvent for this oxidation (Entry 16), while difluoroacetic acid was not (Entry 15). The use of concd sulfuric acid resulted in a formation of a mixture of $\mathbf{3 a}$ and 2-adamantanone in low yields (Entry 17). ${ }^{\text {1a }}$ 


\begin{tabular}{|c|c|c|c|c|c|c|c|c|}
\hline Entry & Substrate & Catalyst & (equiv) & Solvent & Atmosphere & Time (h) & Product 3a-c & Yield $(\%)^{b}$ \\
\hline 1 & $1 \mathbf{a}$ & - & $(-)$ & TFA & $\mathrm{O}_{2}$ & 24 & $3 \mathbf{a}$ & 0 \\
\hline 2 & $1 \mathbf{a}$ & $\mathrm{NaNO}_{2}$ & $(0.01)$ & TFA & $\mathrm{O}_{2}$ & 3 & $\mathbf{3 a}$ & 92 \\
\hline 3 & $1 \mathbf{a}$ & $\mathrm{NaNO}_{2}$ & $(0.2)$ & TFA & $\mathrm{O}_{2}$ & 3 & $3 \mathbf{a}$ & 96 \\
\hline 4 & $1 b$ & $\mathrm{NaNO}_{2}$ & $(0.2)$ & TFA & $\mathrm{O}_{2}$ & 3 & $\mathbf{3 b}$ & 96 \\
\hline 5 & 1c & $\mathrm{NaNO}_{2}$ & $(0.2)$ & TFA & $\mathrm{O}_{2}$ & 3 & $3 c$ & 96 \\
\hline 6 & $1 \mathbf{a}$ & $\mathrm{NOBF}_{4}$ & $(0.2)$ & TFA & $\mathrm{O}_{2}$ & 3 & $3 \mathbf{a}$ & 91 \\
\hline 8 & $1 \mathbf{a}$ & $\mathrm{NaNO}_{2}$ & $(0.2)$ & TFA & air & 3 & $\mathbf{3 a}$ & 92 \\
\hline 9 & $1 \mathbf{a}$ & $\mathrm{NaNO}_{2}$ & $(0.2)$ & TFA & $\mathrm{N}_{2}$ & 3 & $\mathbf{3 a}$ & 10 \\
\hline 10 & $1 \mathbf{a}$ & $\mathrm{NaNO}_{2}$ & $(1.0)$ & TFA & $\mathrm{N}_{2}$ & 3 & $3 \mathbf{a}$ & 46 \\
\hline 11 & $1 \mathbf{a}$ & $\mathrm{NaNO}_{2}$ & $(2.0)$ & TFA & $\mathrm{N}_{2}$ & 3 & $\mathbf{3 a}$ & 92 \\
\hline 12 & $1 \mathbf{a}$ & $\mathrm{NaNO}_{2}$ & $(1.0)$ & $\mathrm{AcOH}$ & $\mathrm{O}_{2}$ & 24 & $\mathbf{3 a}$ & 0 \\
\hline 13 & $1 \mathbf{a}$ & $\mathrm{NaNO}_{2}$ & $(1.0)$ & $\mathrm{MsOH}$ & $\mathrm{O}_{2}$ & 3 & $\mathbf{3 a}$ & 7 \\
\hline 14 & $1 \mathbf{a}$ & $\mathrm{NaNO}_{2}$ & $(1.0)$ & $\mathrm{MsOH}$ & $\mathrm{O}_{2}$ & 72 & $3 \mathbf{a}$ & 89 \\
\hline 16 & $1 \mathbf{a}$ & $\mathrm{NaNO}_{2}$ & $(1.0)$ & $\mathrm{ClCF}_{2} \mathrm{CO}_{2} \mathrm{H}$ & $\mathrm{O}_{2}$ & 3 & $3 \mathbf{a}$ & 93 \\
\hline 17 & $1 \mathbf{a}$ & $\mathrm{NaNO}_{2}$ & $(1.0)$ & concd $\mathrm{H}_{2} \mathrm{SO}_{4}$ & $\mathrm{O}_{2}$ & 24 & $3 \mathbf{a}$ & $31^{c}$ \\
\hline
\end{tabular}

${ }^{\text {a }}$ The reaction was carried out by stirring a solution of substrate $(1 \mathrm{mmol})$ and a catalyst in a solvent $(5 \mathrm{~mL})$ at r.t. under an atmosphere described in the Table.

${ }^{\mathrm{b}}$ Isolated yield after treatment of the reaction mixture with $1 \mathrm{~N} \mathrm{HCl}(10 \mathrm{~mL})$ overnight.

${ }^{\mathrm{c}}$ In addition, 2-adamantanone (18\%) was isolated as a byproduct.

Further information for the mechanism was speculated on the basis of the ratios of the oxidation products in competitive oxidation reactions between $\mathbf{1 a}, \mathbf{1 b}$, and $\mathbf{1 c}$ by $\mathrm{NaNO}_{2}$ and by electrochemical method, ${ }^{2 \mathrm{~m}}$ which has been known to initiate the oxidation of $\mathbf{1 a}$ with electron transfer (Scheme 2). The results are summarized in Table 2 , in which the ratios of products 3a-c almost depended on the number of bridgehead hydrogens of 1a-c in the competitive oxidation reactions by $\mathrm{O}_{2} / \mathrm{NaNO}_{2}$ (Entries 13 ), whereas the competitive oxidation reactions between 1a-c by electrochemical method afforded 3a-c with 1.11.3 ratio of products $\mathbf{3 a - c}$, which were not dependent on the number of bridgehead hydrogens of 1a-c (Entries 4$6)$.

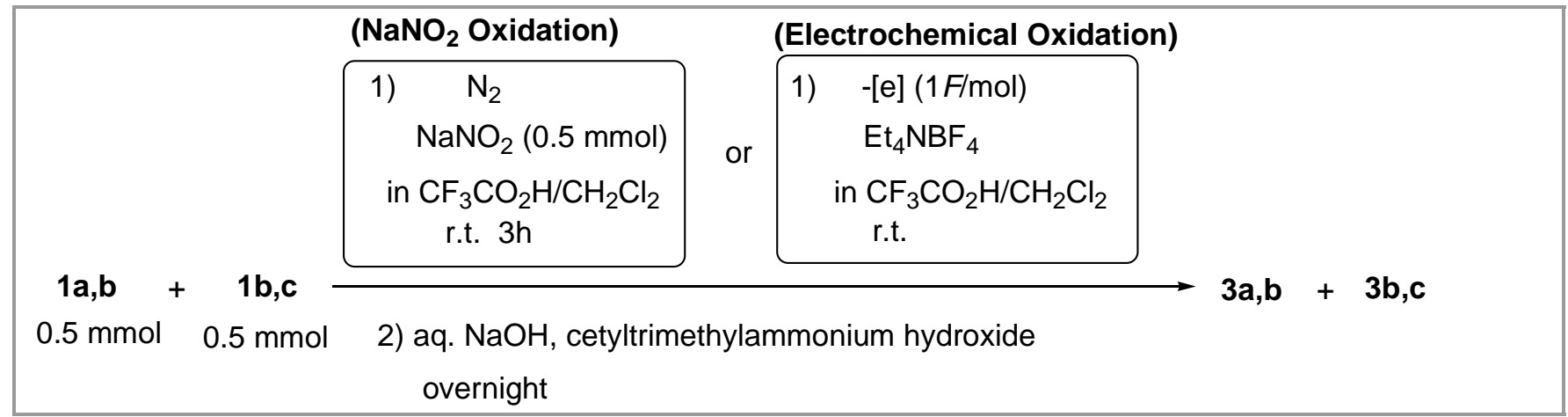

Scheme 2 Competitive oxidation reaction between $\mathbf{1 a - c}$ by $\mathrm{NaNO}_{2}$ and by electrochemical method

An involvement of radical species in those oxidation reactions was then examined by adding TEMPO (2,2,6,6-tetramethyl-1-piperidinyloxy) as a radical scavenger $^{11}$ to a solution for oxidation of 1a. In the oxidation of $1 \mathbf{a}$ by $\mathrm{O}_{2} / \mathrm{NaNO}_{2}$, as an amount of TEMPO increased, the yield of product 3a decreased (Scheme 3), while in the electrochemical oxidation of $\mathbf{1 a}$ addition of 0.2-1.0 equiv of TEMPO didn't show any effect to the reaction (Scheme 4).

On the basis of those results, we propose that NO works as a hydrogen abstractor from 1a to afford adamantyl radical as an intermediate (Scheme 5$)^{12}$ since it has been reported that active species from $\mathrm{NaNO}_{2}$ in TFA for aromatic nitration include NO as shown in Scheme $6,{ }^{13}$ and NO has been known to oxidize dihydropyridine with a hydrogen abstraction mechanism. ${ }^{14}$ This proposed mechanism can be supported by the facts that the competitive reactions between 1a-c depended on the number of bridgehead hydrogens of 1a-c, and that 1 equiv of TEMPO completely retarded the oxidation of 1 a by $\mathrm{O}_{2} / \mathrm{NaNO}_{2}$. On the other hand, electrochemical oxidation of 1a was not affected by TEMPO since it proceeded with electron transfer mechanism. 
NO may also work to oxidize adamantyl radical to adamantyl cation and it can be regenerated by oxygen since 2 equiv of $\mathrm{NaNO}_{2}$ was necessary to complete the oxidation of 1a under a nitrogen atmosphere.

\begin{tabular}{|c|c|c|c|c|c|}
\hline Table 2 & Competitive Reactions be & ween $\mathbf{1 a}, \mathbf{b}, \mathbf{c}$ by $\mathrm{NaNO}_{2}{ }^{\mathrm{a}}$ a & Electrochemical Oxidation ${ }^{\mathrm{b}}$ & & \\
\hline Entry & Substrate $\mathbf{1 a}, \mathbf{b}\left(\mathrm{R}^{1}, \mathrm{R}^{2}\right) / \mathbf{1} \mathbf{b}, \mathbf{c}\left(\mathrm{R}^{1}, \mathrm{R}^{2}\right)$ & $\begin{array}{l}\text { Number of Bridgehead } \\
\text { Hydrogen (Ratio) }\end{array}$ & Oxidation Method & Product $^{\mathrm{c}}$ & $\begin{array}{l}\text { Ratio of Product } \\
\mathbf{3 a}, \mathbf{b} / \mathbf{3 b}, \mathbf{c}\end{array}$ \\
\hline 1 & $\mathbf{1 a}\left(\mathrm{R}^{1}=\mathrm{R}^{2}=\mathrm{H}\right) / \mathbf{1} \mathbf{b}\left(\mathrm{R}^{1}=\mathrm{H}, \mathrm{R}^{2}=\mathrm{Me}\right)$ & $4 / 3(1.3)$ & $\mathrm{NaNO}_{2}$ & $\mathbf{3 a} / \mathbf{3 b}$ & 1.4 \\
\hline 2 & $\mathbf{1 a}\left(\mathrm{R}^{1}=\mathrm{R}^{2}=\mathrm{H}\right) / \mathbf{1} \mathbf{c}\left(\mathrm{R}^{1}=\mathrm{R}^{2}=\mathrm{Me}\right)$ & $4 / 2(2.0)$ & $\mathrm{NaNO}_{2}$ & $3 a / 3 c$ & 2.2 \\
\hline 3 & $\mathbf{1 b}\left(\mathrm{R}^{1}=\mathrm{H}, \mathrm{R}^{2}=\mathrm{Me} / \mathbf{1} \mathbf{c}\left(\mathrm{R}^{1}=\mathrm{R}^{2}=\mathrm{Me}\right)\right.$ & $3 / 2(1.5)$ & $\mathrm{NaNO}_{2}$ & $3 \mathbf{b} / \mathbf{3 c}$ & 1.5 \\
\hline 4 & $\mathbf{1 a}\left(\mathrm{R}^{1}=\mathrm{R}^{2}=\mathrm{H}\right) / \mathbf{1} \mathbf{b}\left(\mathrm{R}^{1}=\mathrm{H}, \mathrm{R}^{2}=\mathrm{Me}\right)$ & $4 / 3(1.3)$ & Electrochemical Oxidation & $\mathbf{3 a} / \mathbf{3 b}$ & 1.1 \\
\hline 5 & $\mathbf{1 a}\left(\mathrm{R}^{1}=\mathrm{R}^{2}=\mathrm{H}\right) / \mathbf{1} \mathbf{c}\left(\mathrm{R}^{1}=\mathrm{R}^{2}=\mathrm{Me}\right)$ & $4 / 2(2.0)$ & Electrochemical Oxidation & $3 a / 3 c$ & 1.3 \\
\hline 6 & $\mathbf{1 b}\left(\mathrm{R}^{1}=\mathrm{H}, \mathrm{R}^{2}=\mathrm{Me} / \mathbf{1} \mathbf{c}\left(\mathrm{R}^{1}=\mathrm{R}^{2}=\mathrm{Me}\right)\right.$ & $3 / 2(1.5)$ & Electrochemical Oxidation & $\mathbf{3 b} / \mathbf{3 c}$ & 1.1 \\
\hline & $\begin{array}{l}\text { action was carried out by stirring a sol } \\
\text { ethane }(3 \mathrm{~mL}) \text { at r.t. under a nitrogen }\end{array}$ & $\begin{array}{l}\text { on of substrates (each } \\
\text { mosphere for } 3 \mathrm{~h} \text {. }\end{array}$ & ol) and $\mathrm{NaNO}_{2}(0.5 \mathrm{mmol})$ & a mixtu & $\mathrm{A}(5 \mathrm{~mL})$ and di- \\
\hline b & $\begin{array}{l}\text { of electricity was passed through a s } \\
\text { eethane }(5 \mathrm{~mL}) \text {. }\end{array}$ & ution of substrates (eacl & $\mathrm{amol})$ and $\mathrm{Et}_{4} \mathrm{NBF}_{4}(1.0 \mathrm{~m}$ & $\mathrm{n}$ a mi & TFA $(5 \mathrm{~mL})$ and \\
\hline
\end{tabular}

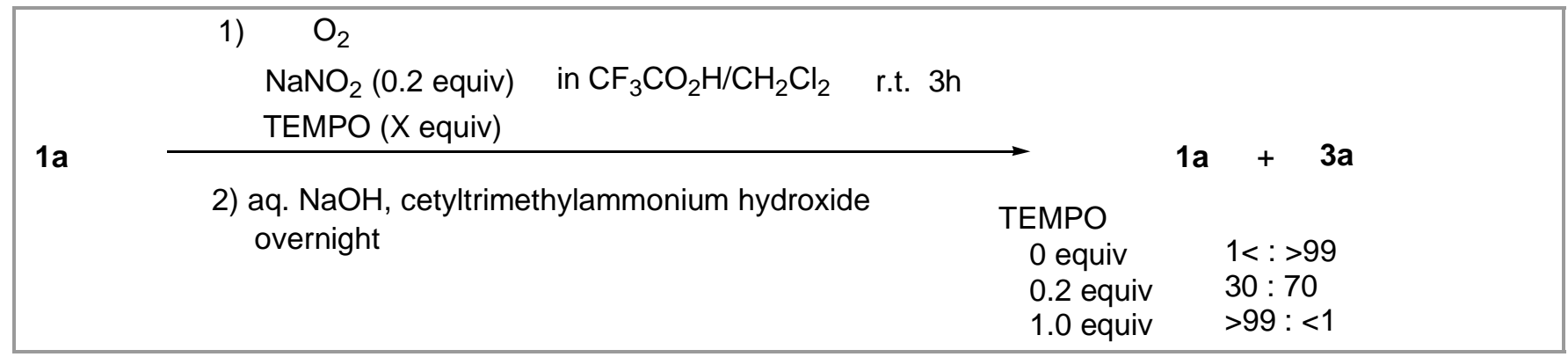

Scheme 3 Influence of TEMPO on the $\mathrm{O}_{2} / \mathrm{NaNO}_{2}$ oxidation of $\mathbf{1 a}$

\begin{tabular}{|c|c|c|c|}
\hline \multirow{2}{*}{$1 a$} & $\begin{array}{l}\text { 1) }-[\mathrm{e}](1 \mathrm{~F} / \mathrm{mol}) \\
\mathrm{Et}_{4} \mathrm{NBF}_{4} \text { in } \mathrm{CF}_{3} \mathrm{CO}_{2} \mathrm{H} / \mathrm{CH}_{2} \mathrm{Cl}_{2} \text { r.t. } \\
\text { TEMPO (X equiv) }\end{array}$ & & $+3 a$ \\
\hline & $\begin{array}{l}\text { 2) aq. } \mathrm{NaOH} \text {, cetyltrimethylammonium hydroxide } \\
\text { overnight }\end{array}$ & $\begin{array}{l}\text { TEMPO } \\
0 \text { equiv } \\
0.2 \text { equiv } \\
1.0 \text { equiv }\end{array}$ & $\begin{array}{l}51: 49 \\
51: 49 \\
52: 48\end{array}$ \\
\hline
\end{tabular}

Scheme 4 Influence of TEMPO on electrochemical oxidation of 1a

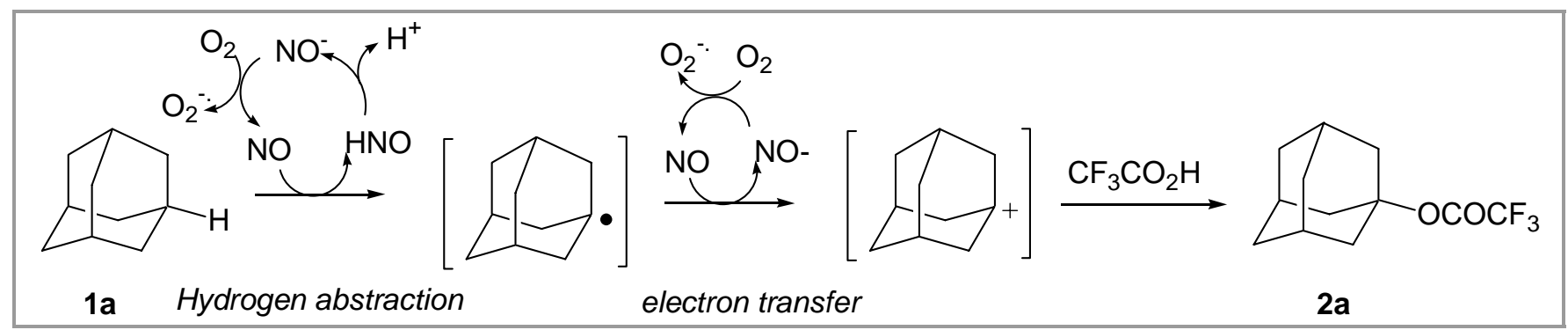

Scheme 5 Plausible reaction mechanism for oxidation of $\mathbf{1 a}$ by $\mathrm{O}_{2} / \mathrm{NaNO}_{2}$ 


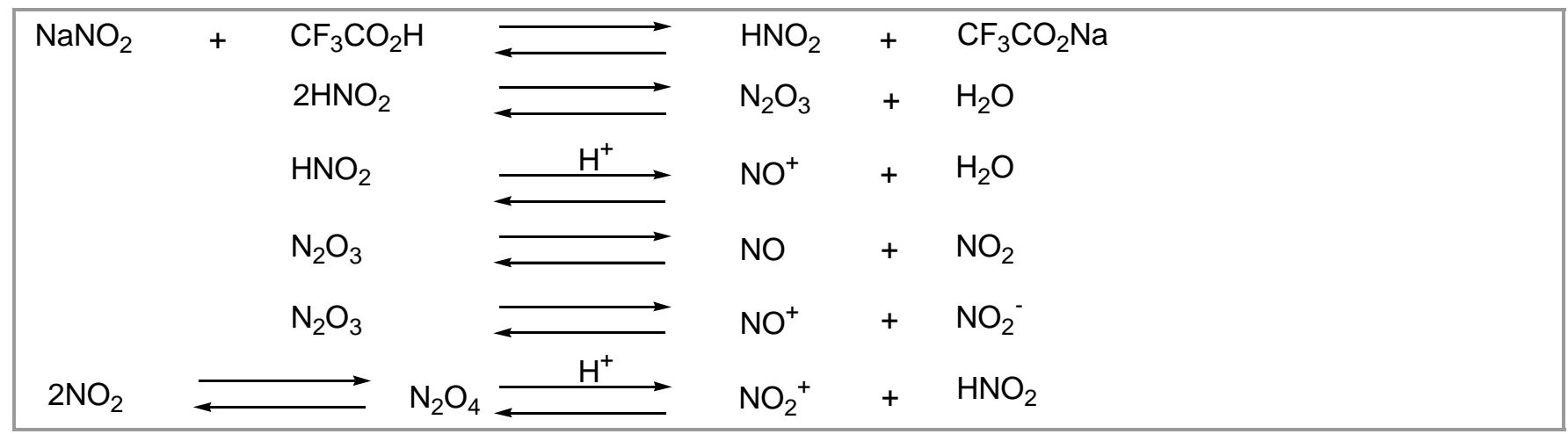

Scheme 6 Active species generated from $\mathrm{NaNO}_{2}$ in TFA

The method presented herein is very promising from a practical viewpoint for oxidation of adamantanes since the reaction conditions are mild, the yields of 3a-c are high, sodium nitrite is an economically available reagent, and most of TFA can be recovered, ${ }^{15}$ though the solvent effect can not be explained at present. Further application of this method to other organic compounds than adamantanes is now under investigation.

\section{References}

(1) (a) Geluk, H. W.; Keizer, V. G. Org. Synth. 1988, Coll. Vol. VI, 48. (b) Krasnikov, S. V.; Obuchova, T. A.; Yasinskii, O. A.; Balakin K. V., Tetrahedron Lett. 2004, 45, 711. (c) Lee, G. S.; Bashara, J. N.; Sabih, G.; Oganesyan, A.; Godjoian, G.; Duong, H. M.; Marinz, E. R.; Gutierrez, C. Org. Lett. 2004, 6, 1705.

(2) For examples, (a) by lead(IV): Jones, S. R.; Mellor, J. M. J. Chem. Soc., Perkin Trans. 1 1976, 2576. (b) by chromium(VI): Carlsen, P. H .J.; Kjaerstad, C.; Aasb $\varphi$, K. Acta Chem. Scand. 1995, 49, 152. (c) by $\mathrm{Re}(\mathrm{VII})$ with $\mathrm{H}_{2} \mathrm{O}_{2}$ : Murray, R. W.; Iyanar, K.; Chen, J.; Wearing, J. T. Tetrahedron Lett. 1995, 36, 6415-6418. (d) by Ru(II) with PhIO: Jitsukawa, K.; Oka, Y.; Einaga, H.; Masuda, H. Tetrahedron Lett. 2001, 42, 3467-3469. (e) by Ru(II) with TBHP: Che, C.-M.; Cheng, K.-W.; Chan, M. C. W.; Lau, T.-C.; Mak, C.-K. J. Org. Chem. 2000, 65, 7996-8000. (f) by Fe(VI); Delaude, L.; Laszlo, P. J. Org. Chem. 1996, 61, 6360-6370. (g) by $\mathrm{O}_{3}$ : Cohen, Z.; Varkony, H.; Keinan, E.; Mazur, Y. Org. Synth. 1988, Coll. Vol. VI, 43-47. (h) by oxone: Kumarathasan, R.; Hunter, N. R. Org. Prep. \& Proc. 1991, 23, 651. (i) by dioxirane: Mello, R.; Cassidei, L.; Fiorentino, M.; Fusco, C.; Curci, R. Tetrahderon Lett. 1990, 31, 3067. (j) by oxaziridine: DesMarteau, D. D.; Donadelli, A.; Montanari, V.; Petrv, V. A.; Resnari, G. J. Am. Chem. Soc. 1993, 115, 4897. (k) by DDQ: Tanemura, K.; Suzuki, T.; Nishida, Y.; Satsumabayashi, K.; Horaguchi, T. J. Chem. Soc., Perkin Trans. 1 2001, 3230. (1) by MCPBA: Takaishi, N.; Fujikura, Y.; Inamoto, Y. Synthesis 1983, 293: (m) by electrochemical oxidation: Bewick, A.; Edwards, C. J.; Jones, S. R.; Mellor, J. M. Tetrahedron Lett. 1976, 631. (n) by $\mathrm{RhO}_{3}$ with $\mathrm{H}_{2} \mathrm{O}_{2}$ in TFA: Nomura, K.; Uemura, S. J. Chem. Soc., Chem. Commun. 1994,129.

(3) Mastrorilli, P.; Nobile, C. F. Tetrahedron Lett. 1994, 35, 4193.

(4) Perutka, J.; Martell, A. E. Anal. Chim. Acta 2001, 435, 385.
(5) Ishii, Y.; Nakayama, K.; Takeno, M.; Sakaguchi, S.; Iwamura, T.; Nishiyama, Y. J. Org. Chem. 1995, 60, 3934.

(6) (a) Germenati, L.; Dondi, D.; Fagnoni, M.; Alibini, A. Tetrahedron 2003, 59, 6409. (b) Ohno, T.; Mitui, T.; Matsumura, M. J. Photochem. \& Photobiol. A: Chem. 2003, 160,3 .

(7) Maki, S.; Konno, K.; Takayama, H. Tetrahedron Lett. 1997, $38,7067$.

(8) Neumann, R.; Dahan, M. J. Am. Chem. Soc. 1998, 120, 11969.

(9) Shinachi, S.; Matsushita, M.; Yamaguchi, K.; Mizuno, N. J. Catal. 2005, 233, 89 .

(10) Olah, G. A.; Ramaiah, P.; Rao, C. B.; Sandford, G.; Golam, R.; Trivedi, N. J.; Olah, J. A. J. Am. Chem. Soc. 1993, 115, 7246.

(11) Dinoi, A.; Curci, R.; Carloni, P.; Damiani, E.; Stipa, P.; Greci, L. Eur. J. Org. Chem. 1998, 871.

(12) The reaction course of $\mathrm{O}_{2}^{-}$described in Scheme 5 is under investigation.

(13) Uemura, S.; Toshimitsu, A.; Okano, M. J. Chem. Soc., Perkin Trans. 1 1978, 1076.

(14) Ito, T.; Nagata, K.; Matsuya, Y.; Miyazaki, M.; Ohsawa, A. J. Org. Chem., 1997, 62, 3582.

(15) Spitzer, U. A.; Stewart, R. J. Org. Chem. 1974, 39, 3936. 
